\title{
On the Photographic Reciprocity Law Failure and Related Effects. I. The Low Intensity Failure
}

\author{
E. KATZ \\ Physics Department, University of Michigan, Ann Arbor, Michigan
}

(Received April 29, 1949)

(1) The order principle (o.p.) states: "The grains of an emulsion become developable in the course of an exposure in a given sequence independent of the exposure and development variables." This hypothesis appears to have a wide range of applicability. It forms an ideally simple link for correlating experimental isodense exposures and theoretical single grain response predictions. Moreover, in many cases where the o.p. is not strictly applicable, corrections can be derived (Section 12) which again make quantitative comparison of theory and experimental data possible.

(2) Extending ideas of Gurney, Mott, and Webb, a quantitative theory of the low intensity reciprocity law failure (l.i.f.) is developed, by analyzing the statistics of light absorption and the survival function of the electrons. Confrontation of various theoretical possibilities with experimental data selects the following basic assumptions: (a) A monoatomic Ag speck of sub-latent image is unstable against thermal agitation, but a diatomic speck is stable for most practical purposes; finally a triatomic speck is completely stable. (b) The energy required to set free an electron from a monoatomic speck (electron trap depth) is not the same for all traps. There must be a quasi-continuous distribution of depths (e.g. as given by formula (10)).

With these assumptions the theory describes satisfactorily the principal features in the shape of experimental l.i.f. curves. It is found that the li.f. slope varies systematically with grain size and the theory checks qualitatively with this result. The experimental temperature dependence of li.f. curves agrees satisfactorily with the theory.

\section{INTRODUCTION}

$T$ HE photographic density $D$ does not depend only on the product $E$ of the intensity $I$ and the time $t$ of exposure. Its dependence on $I$ and $t$ is shown schematically in Fig. 1, using the customary variables $D ; e={ }_{10} \log E ; i={ }_{10} \log I$. Figs. $1 \mathrm{a}$ and $1 \mathrm{~b}$ show the optimal intensity where $E$ is a minimum for given $D$ or $D$ is a maximum for given $E$. To the left and to the right of the optimum lie the regions of "low" and "high intensity reciprocity law failure" (r.l.f.). For the present we shall concentrate on the low intensity failure (li.i.f.). Its existence means that longtime intervals between successive absorptions of quanta in one grain (interquantic times) reduce the efficiency of latent image formation. Optimal interquantic times are usually $\sim 0.01 \mathrm{sec}$. at room temperature.

The present paper aims at a semi-theoretical description of the l.i.f. as a basis for the quantitative treatment of such related effects as the sequence effect (II), the intermittence effect (III) and the addition effect (IV).

\section{ELEMENTARY RELATIONS}

The identity:

$$
(\delta D / \delta i)_{E} \equiv-(\delta D / \delta e)_{I} \cdot(\delta e / \delta i)_{D}
$$

holds at any point of the $D(e, i)$ surface.

The quantity:

$$
\gamma \equiv(\delta D / \delta e)_{I}
$$

is practically constant for a large range of intermediate $D$ values (see Fig. 1c). For given $D$, moreover, $\gamma$ is only very weakly dependent on $I$. Consequently, in Fig. 1a curves with parameter values $D$ and $D+\Delta D$ are practically parallel, their spacing $\Delta e=\Delta D / \gamma$. Similarly, in Fig. 1b curves for $e$ and $e+\Delta e$ are parallel only for intermediate densities where the curves of Fig. 1c are straight.

\section{THE RELATION BETWEEN DENSITOMETRIC AND SINGLE GRAIN DATA}

Experiment generally yields densitometric data whereas theory usually discusses the single grain. How do relations in one field show up in the other one?

The density-exposure curve for a single grain is a step function. Below a certain exposure the grain is not developable but above this it develops completely. The density-exposure curve (Fig. 1c) of the whole emulsion is the sum of a great many of such step functions with different step points and step heights. These curves are sensitive to all spreading effects, such as, e.g., a spread in grain size. Hence they are not well suited to a study of single grain properties.

Do isodense exposures-like those of Fig. 1a-truly represent single grain properties? A. van Kreveld ${ }^{1}$ gave a first answer: If isodensity has an intrinsic physical meaning, the following "transformation law" should hold: "exposures which are isodense on a given emulsion under one set of conditions of development and densitometry should again be isodense on the same emulsion under any other set of such conditions." Experiments proved this to be only very approximately true.

As a new approach to the same problem we propose the following hypothesis (to be called "order principle," o.p.): "The order in which the grains of a given plate become developable in the course of any exposure is independent of the conditions of exposure and development." An equivalent way of stating the o.p. is: "Exposures that would be isodense on one plate by one particular method of development would render the same grains developable." Hence isodense exposures produce exactly equal effects in the last grain(s) rendered developable. Thus the o.p. links in a very simple way experimental isodense exposures and theoretical

\footnotetext{
1 A. van Kreveld, thesis, Utrecht (1933).
} 
single grain data. We shall assume its validity whenever we have no reason to the contrary.

\section{LIMITATIONS OF THE ORDER PRINCIPLE}

There are a number of obvious restrictions to the validity of the o.p.:

(a) Isodense exposures on a panchromatic plate, produced either by blue or red light will not render all the same grains developable. The red light will favor more grains in deeper layers than the blue light. The o.p. cannot obtain then. Unless otherwise stated we will compare only isodense exposures either in thin emulsions or with the same wave-lengths.

(b) If not all grains of an emulsion are sensitized, blue light will affect all grains but red light only the sensitized ones. Isodensity would then refer to different grains and the o.p. does not hold. Unless otherwise stated we shall assume equal absorption spectra for all grains for all wave-lengths concerned.

(c) One may argue that the o.p. can only hold if either the grain size is rather homogeneous or the sensitivity is a monotonous function of grain size and does not spread very much within each grain size group. We shall assume that at least one of these requirements is satisfied.

(d) Suppose the plate contains a mixture of two or more kinds (groups) of grains and the o.p. holds in each group. In general it will not hold for their sum. Some situations where the o.p. does not apply can be resolved into fractions where it does, and in this way can be handled relatively simply (see Section 12 ).

(e) Because of the statistical nature of light absorption, even for two experimentally identical exposures on identical plates, the interquantic times for similar grains will fluctuate. Consequently the place of each grain in the order series may fluctuate. Also other links in the chain of processes of latent image formation may be subject to statistical fluctuations, causing corresponding order fluctuations of the grains. We wish to include this sort of statistical flexibility in the o.p. Such fluctuations will never violate any conclusions based on the o.p. in the stricter sense because most grains will fluctuate only a few places of order and the grains taking their places are on the average very similar in all respects.

Other examples may be constructed, restricting the applicability of the o.p. In practice the error arising from disregarding these restrictions will usually be very small and sometimes can be corrected (see Section 12).

It is not easy to verify the o.p. experimentally. The difficulty in a direct test arises from the fact that any experiment to establish the order in which grains become developable as a result of one type of exposure destroys the possibility of performing a second similar experiment with another type of exposure on the same grains. The o.p. can at present only be tested from its consequences. The present study aims at deriving such consequences and comparing them quantitatively with experiment. The l.i.f. and associated phenomena will appear to be particularly amenable to discussion from the standpoint of the o.p.

\section{THE DIFFERENCE BETWEEN THE ORDER PRINCIPLE AND THE TRANS- FORMATION LAW}

The o.p. may hold without the transformation law and the transformation law may hold without the o.p. One example of each kind will be described:

(a) The o.p. is assumed to hold. Consider two different developers. The first one develops all grains that have surface silver specks of $n$ or more atoms, but ignores grains with smaller specks. The second developer acts on silver specks of $(n-1)$ or more atoms. There is no reason why two different exposures that produce the same number of grains with $n$-atomic specks (i.e., that are isodense with the first developer) should also produce the same number of grains with $(n-1)$-atomic specks (i.e., be isodense with the second developer). In general, therefore, even with the o.p., the isodensity of two different exposures will not be invariant to changes in developer, hence the transformation law is not valid.

(b) The transformation law is assumed to hold. For two isodense exposures, one to red light and one to blue light on a pan plate without r.l.f., the o.p. will not hold because the red light penetrates deeper into the emulsion, consequently involving different grains. The resulting densities, however, are equal for any kind of development.

\section{THE MECHANISM OF THE LOW INTENSITY FAILURE AND ITS CONSEQUENCES}

In order to explain the l.i.f. Gurney and Mott ${ }^{2}$ assumed temporarily that a sulfide sensitivity speck in the silver bromide grain has a tendency to dissociate into an electron and a positive speck owing to thermal agitation. Webb, ${ }^{3}$ on the other hand, explains the li.f. by assuming that a silver speck is unstable against thermal agitation, dissociating into a conduction electron and expelling a $\mathrm{Ag}^{+}$ion into an interstitial lattice position, as long as the speck has not yet surpassed a critical size. From a study of what we shall briefly call the "sequence effect" (see Paper II), Webb derived evidence that this critical size is about half the size required for developability. Webb's hypothesis is in line with known facts about the Herschel effect, where upon absorption of infra-red radiation large (developable) silver specks are known to disintegrate in the same way. Gurney and Mott have apparently accepted Webb's ideas in $1940 .{ }^{4}$

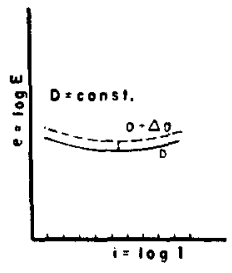

a

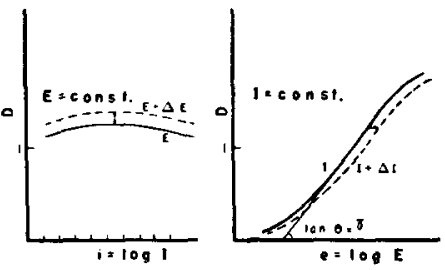

b

c
FIG. 1. The three customary ways of mapping the density $(D)$ $\log$ exposure $(e)$ - $\log$ intensity $(i)$ surface. Fig. $a$ : lines of constant $D$ (r.l.f. curves); Fig. $b$ : lines of constant $e$; Fig. $c$ : lines of constant $i$ ("characteristic" curves).

${ }^{2}$ R. W. Gurney and N. F. Mott, Proc. Roy. Soc. A 164, 151 (1938).

${ }^{3}$ J. H. Webb and C. H. Evans, J. Opt. Soc. Am. 28, 431 (1938).

${ }_{4}$ R. W. Gurney and N. F. Mott, Electronic Processes in Ionic Crystals (Oxford University Press, New York, 1940), p. 247. 
We define an $n$-quantic process as one, the efficiency of which is determined by the time spacings of $n$ quanta. Suppose, for example, that a monoatomic speck of $\mathrm{Ag}$ is unstable against thermal agitation, a diatomic speck is stable and a speck of about 4 atoms is developable. The efficiency of forming a developable speck is determined by the time interval between the quanta that build up the first diatomic speck. The time intervals between later quanta are irrelevant for the efficiency. Hence, this is an example of a two-quantic process in the above sense, although the action of four quanta is required to yield a developable speck. We shall assume that latent image formation is a two-quantic process on the following grounds. It is the simplest assumption consistent with the existence of the l.i.f. The assumption of a triquantic process leads to results that are not supported by experiment (see section 10). The wellknown work of Reinders (recently rediscussed by Berg ${ }^{5}$ ) shows that a developable silver speck contains about 3-4 atoms. The theoretical work of Silberstein ${ }^{6}$ on the shape of characteristic curves also supports the two-quantic assumption.

Now consider one grain absorbing a quantum at $t=0$. Let the survival function $F(t)$ be the chance that at time $t$ there is still, as a result of this absorption, somewhere in the grain an electron "alive." This electron may wander around, being trapped and released at intervals, until it finally "dies" either by being captured in its hole or by being trapped stably at a silver speck. Let $P(t) d t$ be the probability that an interquantic time lies between $t$ and $t+d t$. Then the probability $W$ of liberating a second electron while the first one is still alive is:

$$
W=\int_{0}^{\infty} F(t) \cdot P(t) d t .
$$

If two electrons are present simultaneously, there is a chance $c$ of building a stable speck. Consequently the probability of building a stable speck per interquantic time is $c W$. The probability of forming a stable speck during an exposure with $E^{\prime}$ interquantic times becomes then $\sim\left(1-\exp \left(-c W E^{\prime}\right)\right)$. If $E_{0}$ quanta are needed in addition to render the speck developable, the probability of forming a developable speck during an exposure with $E=E^{\prime}+E_{0}$ interquantic times is $\left(1-\exp \left(-c W\left(E-E_{0}\right)\right)\right)$. Assuming the validity of the o.p., isodense exposures must correspond to the same probability for a developable speck for the type of grains that became developable last. Consequently isodense exposures should satisfy the relation:

$$
W\left(E-E_{0}\right)=\text { const. }
$$

This, then, is also the general expression for the l.i.f. Its explicit form depends on that of $F(t)$ and $P(l)$.

\footnotetext{
${ }^{5}$ W. F. Berg, Phil. Mag. 36, 337 (1948).

${ }^{6}$ L. Silberstein, J. Opt. Soc. Am. 29, 67 (1939).
}

\section{THE STATISTICS OF LIGHT ABSORPTION $P(t)$ AND THE SURVIVAL FUNCTION $F(t)$}

If a grain with effective receptive area $\alpha$ is illuminated with intensity $I$, it absorbs $I \alpha$ quanta per unit time. The average interquantic time $\bar{t}=1 / I \alpha$. It is easily shown (see e.g. $\left.{ }^{7}\right)$ ) that the probability of finding among the fluctuating interquantic times values between $t$ and $t+d t$ is:

$$
P(t) d t=\exp (-t / \hat{t}) d(t / \tilde{t})
$$

With (5), (3) becomes:

$$
W=\alpha I \int_{0}^{\infty} F(t) \exp (-\alpha I t) d t .
$$

For any given $F(t)$ the integral, hence $W$, is a function of $\alpha I$ only.

The present state of knowledge of the interior of the grain does not permit an exact theoretical prediction of the survival chance $F(t)$. If all electron traps had the same potential depth, one would have:

$$
F(t)=\exp (-t / \theta)
$$

This gives with (4) and (6), after a slight rearrangement, for the l.i.f.:

$$
I\left(E-E_{0}{ }^{\prime}\right)=\text { const. }
$$

For very low intensities (7a) approaches:

$$
I E=\text { const. }
$$

A 1.i.f. slope as high as 1 is rarely observed. Mostly the straight part at low intensities satisfies:

$$
I^{x} E=\text { const. with } 0.1<x<0.7
$$

and this corresponds with (4) and (6) to:

$$
I^{x}\left(E-E_{0}\right)=\text { const. }
$$

and:

$$
F(l) \sim t^{-x}
$$

The number of electrons dying per unit time is then:

$$
-d F / d t \sim t^{-x-1} \text {. }
$$

This has to be interpreted in terms of a certain trap depth distribution (see Section 8). The situation in photographic grains may be closely comparable to that in small ZnS-phosphor grains. Antonow-Romanowsky (quoted from Gurney and $\operatorname{Mott}^{4}$ p. 216) studied phosphors of grain sizes only slightly larger than photographic grains. Their decay time constants were roughly of the same order of magnitude as the interquantic times for the li.f. The phosphorescent intensity, which is a direct measure of the number of electrons dying per unit time, was found to be proportional to $t^{-y-1}$ with $0<y<1$. Smaller grains had the smaller $y$ values. Apparently, in small $\mathrm{ZnS}$ grains trap depth distributions do occur which give experimental decay functions such as required to explain the photographic effect.

\footnotetext{
${ }^{7}$ E. Katz, thesis, Utrecht (1941).
} 
This analogy suggests that the slope $x$ of the li.f. should be greater for coarser photographic grains. Table I presents data from literature. Recalling that high speed emulsions have coarser grains than low speed ones we see that the table confirms the expected trend. In addition there seems to be a slight tendency for panchromatic plates to score higher slopes than blue sensitive ones with the same grain size, but more data are needed to ascertain this point.

For the present, then, the form of $F(l)$ has been indicated experimentally.

\section{THE SURVIVAL FUNCTION $F(t)$ AND THE TRAP DISTRIBUTION}

In this section it will be shown that if the grain is assumed to have many traps of various depths for conduction electrons, then by a plausible choice of the depth distribution a survival function $F(t) \sim t^{-x}$, as required by experiment, results. In other words the experimental results can be explained on the basis of a certain trap distribution.

We consider an ensemble of $N$ copies of one grain. Suppose at $t=0$ they all absorb one quantum of light lifting an electron into the conduction band. Let the fraction $m(U) d U$ of all traps in each grain have an energy depth between $U$ and $U+d U$. The form of $m(U)$ is suggested by the following argument. The sensitivity of a grain is mainly due to minute concentrations of $\mathrm{Ag}_{2} \mathrm{~S}$ traps in the $\mathrm{AgBr}$ lattice. Under ideal conditions each $\mathrm{Ag}_{2} \mathrm{~S}$ speck might have the same trap depth. In a real crystal strains, dislocations, cracks in the neighborhood of a speck may vary its trap depth $U$ over a continuous range. Since these physical imperfections themselves constitute (shallow) traps for conduction electrons, their combination with a $\mathrm{Ag}_{2} \mathrm{~S}$ speck will increase the trap depth of the latter. Because the grains have been built and ripened for some time at a temperature $T^{*}$ well above room temperature, the imperfections may have reached equilibrium. Imperfections, the production of which required an energy $V$, will represent a fraction $\exp \left(-V / k T^{*}\right)$ of all the imperfections. The simplest assumption is that the energy depth of a $\mathrm{Ag}_{2} \mathrm{~S}$ trap is increased by an amount proportional to $\mathrm{V}$

TABLE I. Dependence of J.i.f. slope on grain size (experimental).

\begin{tabular}{ccccccc}
\hline Ref. & $\begin{array}{c}\text { Emul- } \\
\text { sion }\end{array}$ & Sensit. & Speed & $\begin{array}{c}\text { Average } \\
\text { grain } \\
\text { in } \boldsymbol{\mu}^{2}\end{array}$ & $\begin{array}{c}\text { 1.j.f. } \\
\text { slope } \\
\boldsymbol{x}\end{array}$ & Radiation \\
\hline 1 & $I$ & pan & high & 0.57 & 0.65 & $4360 \AA$ \\
1 & $I I$ & blue & high & $?$ & 0.5 & $4360 \AA$ \\
1 & $I I I$ & blue & med. & $?$ & 0.23 & $4360 \AA$ \\
2 & $I$ & blue & high & 0.7 & 0.32 & $4360 \AA$ \\
2 & $I I$ & blue & high & 0.7 & 0.25 & $4360 \AA$ \\
2 & $I I I$ & pan & high & 0.5 & 1.0 & $4360 \AA$ \\
2 & $I V$ & blue & low & 0.1 & 0.15 & $4360 \AA$ \\
\hline
\end{tabular}

1 J. H. Webb, J. Opt. Soc. Am. 25, 4 (1935).

2 J. H. Webb, J. Opt. Soc. Am. 23, 159 (1933). owing to a neighboring imper ection. This assumption leads immediately to a distribution:

$$
\left.\begin{array}{ll}
m(U)=0 & \text { for } U<U_{0} \\
m(U)=1 / \epsilon \cdot \exp \left\{\left(U_{0}-U\right) / \epsilon\right\} & \text { for } U \geqslant U_{0}
\end{array}\right\}
$$

where $U_{0}$ is the ideal trap depth of a $\mathrm{Ag}_{2} \mathrm{~S}$ speck and $\epsilon$

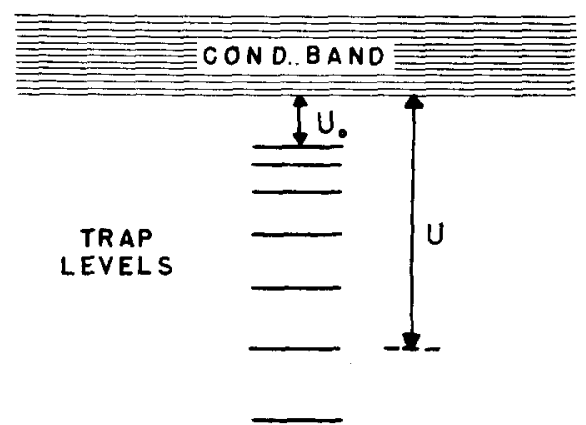

FIG. 2. Schematic distribution of trap depths $U$.

is the average depth increase due to imperfections. It will be shown that the l.i.f. can be described satisfactorily with (10).

Thermal motion tends to liberate a trapped electron. The liberation probability per unit time will be:

$$
\lambda(U)=C \cdot \exp (-U / k T) .
$$

$C$ and an effective depth $U_{e}$ can be estimated by picturing the trapped electron as moving with kinetic energy $\sim k T$ in a potential hole of dimensions $\sim 3.10^{-8}$ $\mathrm{cm}$, the lattice constant. The exponential in (11) represents the escape chance per reflection against the trap wall and $C$ the frequency of hitting the wall. Hence $C=3.10^{14}$. The average time needed for escape must be of the order of the optimal interquantic time $t_{0}(\sim 0.01$ sec. at room temperature). Only if electrons arrive at larger intervals, will previous ones have had a reasonable chance of escaping, dying and thus yielding a l.i.f. Hence :

$$
\lambda_{e} \cdot t_{0} \sim 1
$$

or $\lambda_{e} \sim 100$ and $U_{e} \approx 0.7 \mathrm{ev}$. This value, although derived in a crude way, should be close to all relevant trap depths of the whole distribution since $\lambda$ is very sensitive to changes in $U, 0.1 \mathrm{ev}$ corresponding to a change by factor 60 . For another independent estimate of $U_{e}$ see section 9 .

When an electron escapes from a trap into the conduction band it may recombine with its hole (probability $\gamma$ ) or it may be caught in another trap (probability $\sigma=1-\gamma$ ). Since, in our ensemble, there is at most one electron and one hole per grain, $\gamma$ is a constant. After a time $t$ the fraction $n(U, t, \gamma) d U$ of the ensemble grains will have an electron in traps between $U$ and $U+d U$. The survival function is then:

$$
F(t, \gamma)=\int_{0}^{\infty} n(U, t, \gamma) d U
$$


We have the initial condition:

$$
n(U, 0, \gamma)=m(U)
$$

Traps of depth $U$ may loose electrons by ejection or may gain some that were ejected from other traps. This is expressed by:

$$
\begin{aligned}
& \frac{d n(U, t, \gamma)}{d t}=-\lambda(U) n(U, t, \gamma) \\
& \quad+\sigma m(U) \int_{0}^{\infty} n\left(U^{\prime}, t, \gamma\right) \lambda\left(U^{\prime}\right) d U^{\prime} .
\end{aligned}
$$

A general solution for $n$, hence $F$, in terms of the trap distribution $m$ is not available. We will discuss five cases.

Case $A$ : All traps have the same depth $U_{0} ; \gamma$ arbitrary.

The rigorous solution is:

$$
F(t, \gamma)=\exp \left(-\lambda_{0} \gamma t\right)
$$

Case $B: U$ has a finite number of values $U_{i}$. The deepest traps $U_{m}$ differ from the rest by more than $k T$ or $0.025 \mathrm{ev} ; \gamma$ arbitrary.

The rigorous solution for $F$ is a sum of exponential terms. A short time after the start only one term with $\lambda\left(U_{m}\right)=\Lambda$ in the exponent is of importance:

$$
F(t, \gamma)=c^{\prime} \exp (-\Lambda \gamma c t)
$$

where $c$ and $c^{\prime}$ are of the order of magnitude 1 .

Case C: Arbitrary trap distribution $m(U) ; \gamma=1$; $(\sigma=0)$.

The rigorous solution is:

$$
F(t, 1)=\int_{0}^{\infty} m(U) \exp (-\lambda(U) \cdot t) d U .
$$

If (10) is used for $m$ this becomes for $\lambda_{0} t>1$ :

$$
\text { For } \left.\lambda_{0} t \leqslant 1: \begin{array}{c}
F(t, 1) \approx\left(\lambda_{0} t\right)^{-x} \Gamma(1+x) \approx\left(\lambda_{0} t\right)^{-x} \\
F(t, 1)=1-\left(\lambda_{0} t\right) /(1+x)+\cdots
\end{array}\right\}
$$

where $\lambda_{0}$ refers to $U_{0}$ (see Fig. 2) and $x=k T / \epsilon$. Note that $F(t)$ has here the form (9a).

Case D: Arbitrary $m(U) ; \gamma=1-\sigma$.

No rigorous solution is available, but we can develop $n$, hence $F$, in a power series in $\sigma$ :

$$
F(t, 1-\sigma)=F(t, 1)+G(t) \cdot \sigma+H(t) \cdot \sigma^{2}+\cdots
$$

Here the $p^{\text {th }}$ coefficient can be related through (14) to the $(p-1)^{s t}$ one. The series appears to converge reasonably rapidly. For the distribution (10), excluding very small $t$, one may show:

$$
G(t) \approx g \cdot F(t, 1) \quad(g \leqslant 3)
$$

and $H(t) \ll G(t)$. The principal feature of $F$, its proportionality to $t^{-x}$, remains practically undisturbed for a large range of $\gamma$-values. However, if $\gamma \rightarrow 0$ (case $E$ ) the present approximation ceases to be a good one.

Case E: Arbitrary $m(U) ; \gamma \rightarrow 0$.

For $\gamma \rightarrow 0$ the following reasoning indicates the form of the solution. After a short time during which practically no electrons died, an equilibrium distribution will be reached over the traps. This distribution decays adiabatically, remaining in equilibrium all the time. Under these conditions (14) yields for $F$ in first approximation:

with:

$$
F(t, \gamma \rightarrow 0)=\exp (-\bar{\lambda} \gamma t)
$$

$$
1 / \bar{\lambda}=\int_{0}^{\infty} m(U) / \lambda(U) d U
$$

For a closer approximation we tried:

$$
F(t, \gamma)=\exp (-\bar{\lambda} \gamma t)+\gamma F_{1}(t)+\gamma^{2} F_{2}(t) \cdots
$$

in (14). One finds $F_{1} \equiv 0$. For the distribution (10) it can be shown that for large $t$ the leading term in $F_{2}$ is $\sim t^{-k T / \epsilon}$.

From the analysis of these cases the following conclusions can be drawn for the li.i.f. curve. A small number of trap depths leads invariably to a slope 1 for low intensities according to section 7. This is not in agreement with experiment so that we are driven to the acceptance of a quasi continuous distribution of trap depths. Also in that case extremely small $\gamma$ values still lead to a slope 1 . However, for intermediate and high $\gamma$ values and the distribution (10) the analysis proves that a survival function (9a) as required by experiment results. The slope is then $k T / \epsilon$, according to $(16 \mathrm{c}, \mathrm{d})$. The fact that this slope does not depend on the value of $\gamma$ within a wide range lends credence to the theory. We have no way of measuring $\gamma$ accurately and no theoretical means of estimating it. The theory in this form agrees with experiment. In the next sections further consequences will support this conclusion.

We terminate this section with a few remarks about the value of $k T / \epsilon$. Formula (10) has a high energy tail that is certainly incorrect. No levels can be expected for energies $U>U_{m}$, defined by:

$$
\int_{U_{m}}^{\infty} m(U) d U=1 / M
$$

or:

$$
U_{m}-U_{0}=\epsilon \ln M
$$

where $M$ is the total number of traps per grain. Now the most relevant traps for the l.i.f. are the deepest ones which keep the electrons trapped during the longest times. This may formally be seen from (16e). If the upper limit of integration would be $\infty, \bar{\lambda}$ would diverge. With $U_{m}$ as an upper limit we obtain:

$$
\bar{\lambda} \approx(\epsilon / k T-1) C \exp \left(-U_{m} / k T\right) \approx \lambda\left(U_{m}\right) .
$$

The ripening and manufacture of a plate apparently is conducted in such a manner that the maximum trap 
depth is not too great, which would cause fog. This limit seems to correspond to $\bar{\lambda} \sim 100$ at room temperature. Using this with (17), (18), $C=3.10^{14}$ and $U_{0} \ll U_{m}$ (see Fig. 2) we obtain for the l.i.f. slope:

$$
k T / \epsilon=(\ln M) / 29
$$

General solid state evidence suggests that the relative number of imperfections in a photographic grain may be $\sim 10^{-5}$. For an average grain with $10^{10}$ ion pairs this means $M \sim 10^{5}$. Hence, with (19): $k T / \epsilon=0.40$, which agrees well with average slopes observed. Moreover, (19) indicates why larger grains have steeper slopes (see Table II). The relative amount of imperfection is seen to be not a critical parameter. The qualitative agreement between this table and the experimental data of Table $I$ is as good as may be expected.

\section{THE TEMPERATURE DEPENDENCE}

The influence of the temperature on the l.i.f. consists of a change of the slope and a shift of the line as a whole:

(a) The change of slope. For $\gamma \rightarrow 0$ the slope should $\mathrm{be}=1$, independent of temperature. For intermediate and high $\gamma$-values and the distribution (10) the slope was $k T / \epsilon$, according to $(16 \mathrm{c}, \mathrm{d})$, so that a weak dependence on temperature is expected. The author is not aware of sufficiently accurate data, extending to low enough intensities, permitting a check of this temperature dependence. Such experiments would involve extremely long exposure times because the r.l.f. curves shift as a whole to the low intensity side for lower temperatures. The best data available are those by Webb. ${ }^{8}$ Taking their accuracy into account, they are not in disagreement with any weak slope dependence.

(b) The shift of the curve as a whole. Two isodense points with equal values of $E-E_{0}$ but different $T$ must have the same $W(I)$ according to (4). A pair of such points on neighboring r.l.f. curves will have a difference $\Delta \ln I$ such that:

$$
\begin{aligned}
\Delta \ln W=\partial \ln W / \partial \ln I & \cdot \Delta \ln I \\
& +\partial \ln W / \partial \ln (1 / k T) \cdot \Delta(1 / k T)=0 .
\end{aligned}
$$

Using (6), (9), (9a), (11) and (16c) we obtain:

$$
-\Delta \ln I / \Delta(1 / k T)=U_{e}+k T\left(\ln \lambda_{e} t_{0}+\ln I_{0} / I\right) .
$$

If we compare data at an intensity $I=I_{0} / 100$ and use (11a) the right hand side of (20) becomes $=U_{e}+0.1 \mathrm{ev}$. This result is accurate to the extent to which $\lambda_{e} t_{0}=1$. This is only an order of magnitude relation, so the product may be off by a factor of say 10 , but this affects the result only by $0.05 \mathrm{ev}$. So we see that the temperature shift of the r.l.f. yields a reasonably accurate measure of the effective trap depth $U_{e}$. For $\gamma \rightarrow 0$ the result turns out to be very similar. In comparing (20) with experiment one precaution must be

\footnotetext{
${ }^{8}$ J. H. Webb, J. Opt. Soc. Am. 25, 4 (1935).
}

observed. The optimal exposures are also slightly temperature dependent. This means that only a temperature dependent fraction of the intensity is photo effective. ${ }^{*}$ In order to correct for this effect the r.l.f. curves should be moved as a whole in the direction of lines of constant exposure times, that is in the usual representation towards north-east, until all optimal exposures are equal. If this is done the proportionality between $\Delta \log I$ and $\Delta T$ appears satisfactorily. Table III shows results derived from Webb's quoted paper. ${ }^{8}$ Comparing Table III with formula (20) we conclude that $U_{e}=0.74-0.1=0.64 \mathrm{ev}$. This value is less than the energy of the Herschel effect (1-1.4 ev.) as it should be and is in reasonable agreement with the value of $0.7 \mathrm{ev}$. estimated in section 8 . It suggests that low intensity latent image formation should be greatly slowed down by simultaneous infra-red radiation of wave-length $\sim 2 \mu$.

\section{TRIQUANTIC AND HIGHER ORDER PROCESSES}

In section 6 we postulated that a monoatomic speck is unstable but a diatomic one is stable. Let us investigate briefly the consequences of a theory in which diatomic specks are also unstable but triatomic specks are stable. By arguments similar to those traced out for two quantic processes the equation for the r.l.f. curve

TABLE II. Dependence of l.i.f. slope on grain size (theoretical).

\begin{tabular}{llll} 
Grains & $\begin{array}{c}\text { Ion pairs } \\
\text { per grain }\end{array}$ & $\begin{array}{c}\text { l.i.f. slope according to (19) as- } \\
\text { suming a rel. concentration } \\
\text { of imperfections of: } \\
10^{-5}\end{array}$ & $10^{-6}$ \\
small & $10^{7.5}$ & 0.20 & 0.12 \\
medium & $10^{9}$ & 0.31 & 0.24 \\
large & $10^{10.5}$ & 0.43 & 0.34 \\
\hline
\end{tabular}

TABLE III. The shift of l.i.f. curves with temperature.

\begin{tabular}{lccc}
\hline Emulsion & $\begin{array}{c}\text { Radiation } \\
\AA\end{array}$ & $\begin{array}{c}\text { Temperatures } \\
{ }^{\circ} \mathrm{C}\end{array}$ & $\begin{array}{c}\text { Observed } \\
-\Delta \ln I / \Delta(1 / k T) \text { in ev. }\end{array}$ \\
\hline$I$ & 4360 & $0-50$ & 0.72 \\
$I I$ & 4360 & $0-50$ & 0.71 \\
$I I I$ & 4360 & $0-50$ & 0.77 \\
$I$ & 3650 & $0-50$ & 0.78 \\
$I$ & 5460 & $0-50$ & 0.73 \\
& & & $0.74=$ average \\
& & & 0.82 \\
$I$ & 4360 & $-10-00$ & $0-20$ \\
$I$ & 4360 & $0-50$ & 0.62 \\
$I$ & 4360 & $20-50$ \\
\hline
\end{tabular}

* The cause of this dependence may be as follows: An absorbed quantum first excites an electron and this exciton has a certain probability of dissociating into a free conduction electron and a hole. The probability of dissociation is slightly dependent on temperature, as is known from measurements on photo-conductivity. 


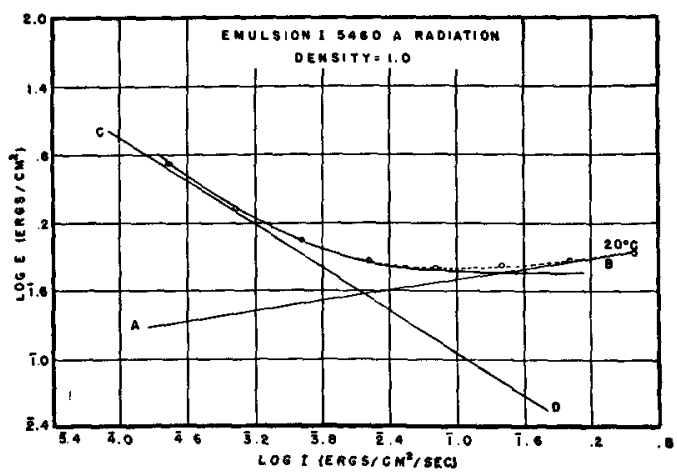

Fig. 3. Theoretical l.i.f. curve (full drawn) adapted to Webb's data (dashed curve and points) and straight line components proposed by Webb.

is again of the form $W \cdot\left(E-E_{0}\right)=$ constant, where now :

$$
\begin{aligned}
W=C /(\bar{t})^{2} \cdot \int_{0}^{\infty} F_{1}(t) \exp (-t / \hat{t}) d t \\
\cdot \int_{0}^{\infty} F_{2}(t) \exp (-t / \bar{t}) d t .
\end{aligned}
$$

The result then depends again on the assumptions regarding the functions $F$, which now have the meaning: $F_{1}=$ the survival chance of one electron till the second one arrives, $F_{2}=$ the survival chance of two electrons till the third one arrives. If exponentials are taken for these functions, the l.i.f. slope becomes 2 . If we assume that one silver atom is very unstable, two silver atoms are metastable and three atoms are practically completely stable, $F_{2}$ has a greater time constant than $F_{1}$. If the activation energy of a monoatomic speck is about $0.7 \mathrm{ev}$. as before, the activation energy of a diatomic speck must lie somewhere between 0.7 and 1-1.4 ev., the latter being the energy of the Herschel quanta, needed for the dissociation of completely stable latent image specks. A value of $0.9 \mathrm{ev}$. seems appropriate for the potential depth of a diatomic speck. The dissociation of a diatomic speck would then be slower by a factor of the order $10^{4}$ than for a monoatomic speck. This simple reasoning suggests, that for intensities, corresponding to interquantic times $t_{0}<t<10^{4} t_{0}$ the r.l.f. curve is still determined only by the two-quantic process of the instability of monoatomic specks. Slopes greater than 1 are not to be expected in this intensity region. For intensities $\sim 10^{4}$ times lower than the optimum one might observe a kink in the r.l.f. curve towards a steeper slope. This slope should be less than 2 if it corresponds to stable triatomic silver specks. For still lower intensities again a kink may occur, toward a still higher slope corresponding to tetratomic specks, the triatomic ones now being unstable for such tremendouly long interquantic times.

The exposure times required to observe the first mentioned kink may be of the order of $10^{6}$ seconds or several days, which is feasible. For the second kink they would be of the order of at least $10^{9}$ seconds which is beyond the experimental range. In trying to verify the predicted behaviour at intensities near and below the first kink the present theory encounters some diffculty. A few experimental data are available down to very low intensities, for instance those of Arens and Eggert ${ }^{9}$ and a kink is then observed. However, the slope of the r.l.f. curve there becomes infinite. The present author is inclined to interpret this too steep upturn as a spurious effect, masking the true kink that would lie at a slightly lower intensity. Namely, no survival function $F$ of the time only exists, which, when used in (6) will cause the observed upturn towards a vertical asymptote, neither is there a pair for (21). Since (6) was based only on very general features of the picture of Webb, Gurney, and Mott, this discrepancy means that either the explanation of the li.f. is basically wrong or that spurious effects veil the true situation in this particular case. ${ }^{* *}$ In view of the agreement of the theory with experiment in so many details it seems reasonable to ascribe the too steep upturn to an unaccounted complication and to withhold conclusions in this region of extremely low intensities until more data are available. With this restriction we may conclude from the evidence presented that the l.i.f. can be described by one survival function and stable diatomic $\mathrm{Ag}$ specks.

\section{THE TRANSITION TO THE REGION OF OPTIMAL INTENSITIES}

According to the present theory the r.l.f. curve as it comes down from the region of low intensities, should bend towards horizontal in the region of optimal intensities. This should be so even if the high intensity failure would be absent, because there must be a minimum number of quanta to produce a developable speck. Most experimental curves soon bend upward owing to the high intensity r.l.f. It is not yet possible to predict how much the high intensity effect makes itself felt at intensities slightly below the optimum, but the following estimate shows that its influence vanishes rapidly with decreasing intensities. Suppose the first electron needs on the average a neutralization time $\tau$ before a second electron can be admitted to the same speck. If the average interquantic time used is $t$ then, according to (5), the chance that another electron will be produced before $\tau$ is $\sim \tau / \bar{t}$ for low intensities (high values of $\bar{t}$ ).

The number of rejections is thus $\sim 1 / \bar{t}$ or $\sim I$, so we may expect that the loss in efficiency is also $\sim I$ and

${ }^{9}$ H. Arens and J. Eggert, Zeits. f. Wiss. Phot. 27, 79 (1929-30),

** Possibly such a spurious effect may be caused by a back reaction of the following kind. The experiments were done with non-monochromatic light. As the efficiency of the active light decreases with lower intensity, the destruction of the $\mathrm{Ag}$ specks by radiation of $2 \mu$, whose efficiency is not reduced, might have become predominant. Destruction of the sub-latent image by a radiation component would make $F(t)$ in (6) dependent on the intensity $I$. Functions $F(t, I)$ causing the observed vertical upturn do exist. 
hence the number of extra electrons needed to reach the same probability of developability is also $\sim I$. This approximation is only valid if we may neglect the chance that more than one electron will arrive in $\tau$. Therefore, the fact that for intensities above optimal the effect appears to go more slowly than $\sim I$ is not in contradiction with the proportionality to $I$ at lower intensities. From this estimate we may conclude that the high intensity component vanishes rapidly below the optimum which justifies neglecting it there.

The bending towards the horizontal will then be assumed to follow formula (9), i.e., $W(I) \sim I^{x}$. This implies that for $E-E_{0}$ the approach to the horizontal in the conventional $e(i)$ plots is exponentially.

Previous empirical formulas describe the r.l.f. curve in the neighborhood of the optimum by a sum of two exponentials. ${ }^{8}$ Hence the present theoretical formula will do well anywhere below the optimum. Checks have been carried out in a number of arbitrary examples and proved to be completely satisfactory. A typical example is given, Fig. 3, representing observations by Webb. ${ }^{8}$ The points are the same ones that were used by Webb for his analysis of the curve into two straight components (p. 17, Fig. 12).*** The fully drawn curve represents formula (9) with $x=0.800$; $E_{0}=0.552$ and the constant $=6.91 \cdot 10^{-3}$, chosen in order to give the best fit. It is evident from this and similar examples that the factor $E-E_{0}$ in (9) gives a very good description of the course of the li.f. right up to the optimum.

In addition the following factors may be of slight influence on the form of the curves:

(a) In the derivation of formula (6) $E$ was assumed to be a constant for a given exposure time and intensity. However, $E$ will also fluctuate, although much less than the individual interquantic times. A certain average is represented by the experimental curve, which, in general, will not be exactly the same as a curve belonging to a non-fluctuating average value of $E$. However, because of the weak curvature of the r.l.f. curve this approximation causes only a negligible error.

(b) Non-uniform light intensity throughout the emulsion and a spread in grain size will cause systematic deviations from the order principle and minute distortions of the r.l.f. curve. They will be treated in Section 12.

(c) It might seem as if the adoption of (9) for the region near the optimum implies the arbitrary assump-

\footnotetext{
*** A purely empirical method of analysis for the entire r.l.f. curve, assuming it to be equal to the sum of two straight lines, was suggested by Webb in 1935 (see reference 8). This method, besides lacking theoretical background, is open to the following fundamental criticism. The two processes represented by the two straight lines could either operate in parallel or in seiies. In parallel this would mean that a small fraction of the developable grains is produced at very low intensities with an abnormally high effciency. If they are in series, it would mean that the partial process of either one occurs at abnormal efficiencies, either at very low or at very high intensities. It is true that the Gurney-Mott picture was not yet available when the straight line analysis was proposed.
}

tion that $W(I)$ remains proportional to $I .^{* * *}$ This may seem too simple. However, the good agreement between theory and experiment regarding the approach to the optimal point comes essentially from the factor $E-E_{0}$ and is very insensitive to the particular choice of $W(I)$. For this reason it seems justified to choose a mathematically simple form for $W(I)$, like (9). For very low intensities where $E-E_{0}$ is a large fraction of $E$, this approximation approaches a straight line that can be given the correct slope and the correct first-order deviation from a straight line. For high intensities it can be given a horizontal asymptote at the correct height. In this manner, by adapting $x, E_{0}$ and the constant an excellent fit to experimental data can always be obtained. For higher intensities $E-E_{0}$ is only a small fraction of $E$ so that the experimental error in $E$ corresponds to a large uncertainty in $E-E_{0}$. Hence any reasonable approximation for $W(I)$ is satisfactory in this region.

\section{THE INFLUENCE OF DEVIATIONS FROM THE ORDER PRINCIPLE}

Let us see what corrections must be applied to our previous statements because of deviations from the o.p. in normal r.l.f. experiments. These corrections will also be useful in subsequent papers.

In order to discuss the matter from a general standpoint we assume that the grains can be divided into groups, indicated by a parameter $a$ (if necessary a may stand for several parameters), such that in each group the o.p. holds. The validity of the o.p. in each group does not imply its validity for the whole plate (see section 4.d). The main corrections arise from the variation of light intensity throughout the emulsion-depth, and the spread in grain size. In both cases the grains can be divided into such groups. If the plate receives an exposure $E$ with intensity $I$, each group will give rise to a partial density $D(a, e, i) d a$ and since densities are known to be additive, the total density is:

$$
D=\int D(\alpha, e, i) d \alpha
$$

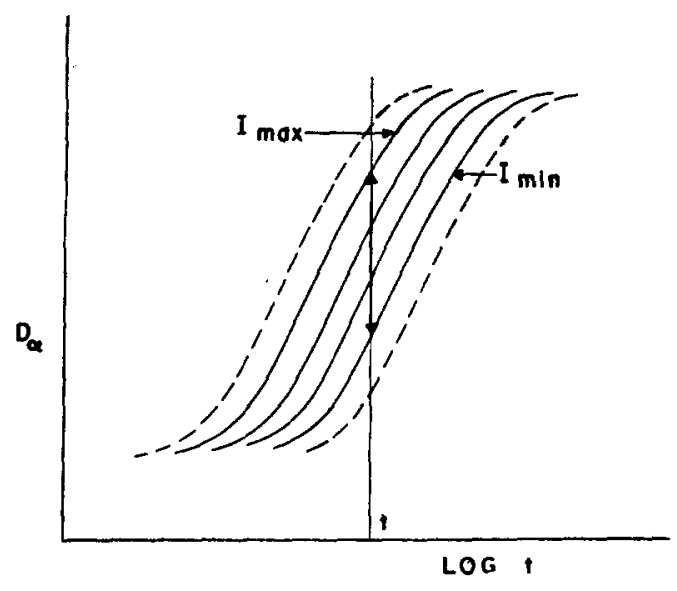

Fig. 4. Illustrating the conditions that $\gamma_{\alpha}$ be constant. 


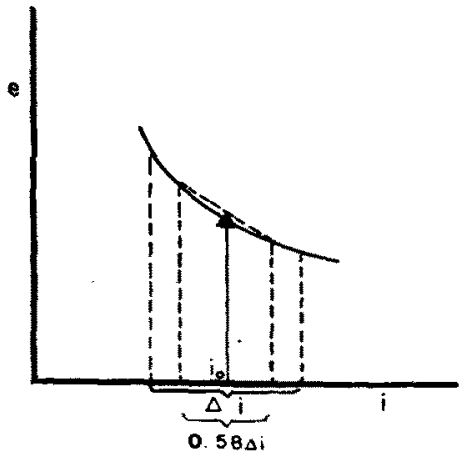

FrG. 5. The influence of non-homogeneous light intensity on the shape of the li.f. curve. According to formula (23) the ordinate at $i_{0}$ is displaced to the center of the chord subtending $0.58 \Delta i=\Delta i / \sqrt{3}$

We wish to find how the shape of total isodense r.l.f. curves compares with the shapes of isodense r.l.f. curves of the various a groups. For this purpose we vary $e$ and $i$, keeping $D$ constant. We abbreviate as in (2):

and observe:

$$
\partial D(a, e, i) / \partial e=\gamma_{\alpha}
$$

$$
\int \gamma_{\alpha} d \alpha=\gamma
$$

Setting $\delta D=0$, we have with (1):

$$
(\delta e / \delta i)_{D}=1 / \gamma \cdot \int \gamma_{\alpha}(\delta e / \delta i)_{D(\alpha)} d \alpha
$$

In words: The observed r.l.f. slope is a certain average of the slopes of the groups. In particular, if all groups have the same slope for a certain value of $i$, the total r.l.f. has there the same slope.

We shall apply this first to the variation of light intensity with depth. Let a stand for the depth in the emulsion. The following assumptions are made:

(a) All grains are of equal size and the undeveloped grains are homogeneously distributed throughout the depth of the emulsion.

(b) $\gamma_{\alpha}$ is independent of $\alpha$.

(c) The light intensity falls exponentially with the depth $\alpha$, bence $i$ is linear with $\alpha$.

Assumption (b) is illustrated in Fig. 4, representing schematically a set of characteristic curves for a single layer with slope $\gamma_{\alpha}$. For an exposure on a thick emulsion the densities to be added lie on a straight vertical segment, corresponding to the exposure time used, and to the intensity range that is present in the plate from front to back. $\gamma_{\alpha}$ will be constant through out the emulsion provided the partial density $D(a)$ lies for the whole plate in the straight range of the corresponding curves and the intensity range through the plate does not cover too large an interval, which is usually true for curves referring to a total density of the order 1 .

With these assumptions (22) yields by straightforward calculation the following results:

In first approximation the r.l.f. curve for the real (inhomogeneous) exposure is the same as that for an ideal (homogeneous) exposure on the same plate, provided the coordinates $e$ and $i$ are interpreted as the values at the center (half-depth) of the plate. Since usually the plots are made with those variables referring to incident values the curve should be shifted horizontally and vertically over a distance $\log I$ (center)/ $I$ (incident).

In second and third approximations the form of the real curve is slightly changed as compared to the ideal one. Straight parts remain straight with the same slope, but curved parts are smoothed out a little. To find the point on the experimental curve with abscissa $i$, one has to take the average of the ordinates of the two points on the ideal curve with abscissae $i \pm \Delta i / 2 \sqrt{3}$ $=i \pm 0.29 \Delta i$, where $\Delta i$ is the log light range from the front to the back of the plate. With $i$ and $e$ again referring to the half-depth we have:

$$
e(i)_{\mathrm{real}}=\frac{1}{2}(e(i+0.29 \Delta i)+e(i-0.29 \Delta i))_{\mathrm{idea3},},
$$

if differences of the order $(\Delta i)^{4}$ and higher are neglected.

For a light range of a factor 10 from front to back, or $\Delta i=1$, as is often the case in practice, the curve for the actual plate should be obtained from the curve for homogeneous light intensity by averaging the values at $i \pm 0.3$. Inspection of actual r.l.f. curves (see e.g. Fig. 3) shows that this correction of the shape is negligible.

We shall next apply (22) to the case of inhomogeneous grain size. Let $a$ represent the effective projective area of the grain. The following assumptions are made:

(a) All grains are exposed to the same intensity.

(b) $\gamma_{\alpha} d \alpha$ is proportional to the saturation density of the size group $\alpha$, hence to the number of grains $N(\alpha) d \alpha$ in this group times the area of the grains $\alpha: \gamma_{\alpha}=c N(\alpha) \alpha$.

(c) The plot of $0.43 N(\alpha) \cdot \alpha^{2}$ (that is the total area of grains between $\log \alpha$ and $\log \alpha+d \log \alpha$ ) versus $\log \alpha$ has a maximum for a certain grain size $\alpha=\alpha_{0}$ and is symmetrical about this maximum. This is the case in most actual emulsions.

(d) $(\partial e / \partial i)_{\log \alpha}=(\partial e / \partial i)_{\log \alpha_{0}}+f(i) \cdot\left(\log \alpha-\log \alpha_{0}\right)$, where $f(i)$ is a small coefficient dependent on $i$, which is justified by the weak dependence on $\alpha$ discussed in section 7 .

With these assumptions (22) yields by straightforward calculations the following results:

In first approximation the r.l.f. curve for the real emulsion is the same as that for an ideal plate containing only grains of class $a_{0}$, with the same total projective area.

In second and third approximations the r.l.f. curve differs slightly in form from that for the ideal $\alpha_{0}$ plate. Straight parts remain straight with the same slope, but curves are smoothed out a little. To find the point with abscissa $i$ for the real plate one has to take the average of the ordinates of the two points on the $a_{0}$-curve with abscissae $i \pm\left\{\left\langle(\Delta \log a)^{2}\right\rangle_{A V}\right\}^{\frac{1}{3}}$ :

$$
\begin{aligned}
e(i)_{\mathrm{real}}=\frac{1}{2}\left[e\left(i+\left\langle(\Delta \log \alpha)^{2}\right\rangle_{A v}{ }^{\frac{1}{2}}\right)\right. & \\
& \left.+e\left(i-\left\langle(\Delta \log \alpha)^{2}\right\rangle_{A v}{ }^{3}\right)\right]_{\alpha_{0}}
\end{aligned}
$$

with:

$$
\left\langle(\Delta \log \alpha)^{2}\right\rangle_{A v} \equiv \int(\Delta \log \alpha)^{2} N(\alpha) \alpha d \alpha / \int N(\alpha) \alpha d \alpha .
$$


Differences of the order $\left\langle(\Delta \log a)^{4}\right\rangle_{A v}$ or higher are neglected.

For many emulsions the function $N(a)$ is known and permits the calculation of $\left\langle(\Delta \log a)^{2}\right\rangle_{\mathrm{Av}}$. For most commercial emulsions this quantity turns out to be $\sim 0.4$. Inspection of r.l.f. curves shows that the error caused by neglecting this second order correction is negligible. In the worst cases at the most curved parts of the r.l.f. curve it amounts to less than 5 percent of exposure. Although the correction is insignificant for the shape of the r.l.f. curve it becomes important for the intermittency effect (see paper III) when small differences in r.l.f. are the object of the theory.

Summarizing we have found that the two main causes for deviations from the o.p., namely inhomogeneous light intensity and grain size, cause the real r.l.f. curve to be practically identical with the ideal r.l.f. curve that would be obtained if the light intensity would be homogeneous and equal to the value at the center of the plate, and if the plate contained only grains of size $a_{0}$, defined above.

Finally we wish to point out that it is not consistent with the observed behaviour of characteristic curves for various intensities, to explain the slope $0<x<1$ of the r.l.f. curves as the result of two groups of grains, the first group having a slope 0 (no r.l.f.) and the second group having a slope $=1$ (one trap depth only), in the intensity region studied. If the o.p. would hold within each one of these two groups the resulting slope would lie indeed between 0 and 1 according to (22), but the characteristic curves for low intensities behave experimentally in a way that contradicts such an assumption. For this reason the scheme with many trap depths had to be introduced.

Note added in proof: The author is greatly indebted to Dr. J. H. Webb for bringing to his attention a series of recent studies by P. C. Burton and W. F. Berg (Phot. J. 86B, 2, 62 (1946) and 88B, 13, 84, 123 (1948)) which provide strong support for the assumptions made in Sections 6 and 11 .

\title{
Dielectric Behavior of Solutions of Electrolytes in Solvents of Low Dielectric Constant. V. The Influence of Temperature and Concentration on Dielectric Properties*
}

\author{
H. A. StrobeL** AND R. H. Cole \\ Metcalf Research Laboratory, Brown University, Providence, Rhode Island
}

(Received April 22, 1949)

\begin{abstract}
Dielectric loss and conductance data for octadecyltributylammonium thiocyanate in benzene show large changes in the significant frequency region of absorption and in the magnitude of conductance when the temperature is lowered from $25^{\circ}$ to $6^{\circ} \mathrm{C}$, but their variation with concentration is very similar at the two temperatures. The broad frequency range of absorption observed at concentrations in the range $0.008-0.02$ molal and the failure of the change in macroscopic viscosity to account for the temperature effect suggest that a better understanding of similar systems requires more detailed examination of the interaction of the ionic and dipolar aggregates with the solvent.
\end{abstract}

$\mathrm{O}^{\mathrm{s}}$ $F$ the several approaches employed in this laboratory in the investigation of the behavior of electrolytes in solvents of low dielectric constant, the measurement of dielectric absorption by a calorimetric method has been among the most revealing. In the earlier papers of this series the information obtained from such measurements has been used, in conjunction with conductance, cryoscopic, and polarization data, to demonstrate the strong dependence of the nature and extent of ion association on the constitution and type of electrolyte., ${ }^{1,2}$

An investigation of the temperature dependence of

* This work was supported by a grant from the Research Corporation.

** Present address: Dept. of Chemistry, Duke University, Durham, North Carolina.

'(a) Sharbaugh, Schmelzer, Eckstrom, and Kraus, J. Chem. Phys. 15, 47 (1947); (b) Sharbaugh, Eckstrom, and Kraus, J. Chem. Phys. 15, 54 (1947).

${ }^{2}$ H. A. Strobel and H. C. Eckstrom, J. Chem. Phys. 16, 817 (1948). the dielectric absorption of such systems in the $5-25^{\circ} \mathrm{C}$ range has formed the basis of the present work. The study was prompted principally by the continued need for new approaches to the complex picture presented by the known results. The available data have been used as well, insofar as seemed justified, in examining the role of concentration as an influence on association. A further result of the work has been the possibility of direct intercomparison of cryoscopic and absorption data.

For the study, octadecyltributylammonium thiocyanate, a fairly strong electrolyte representative of the quaternary ammonium salts already examined ${ }^{3}$ was selected. Benzene solutions of that salt have now been investigated at several concentrations both at $25^{\circ}$ and at about $6^{\circ}$. Audiofrequency conductance determina-

\footnotetext{
${ }^{3}$ With the exception of the picrates, all of the strong quaternary ammonium electrolyte already examined at $25^{\circ}$ have behaved similarly.
} 